\title{
Fuzzy Model for Parameterized Sign Language
}

\author{
Sumaira Kausar \\ Department of Computer Science \\ BahriaUniversity, Islamabad \\ Pakistan
}

\author{
Samabia Tehsin \\ Department of Computer Science \\ Bahria University, Islamabad \\ Pakistan
}

\author{
M. Younus Javed \\ HiTec University, HIT Taxila \\ Pakistan
}

\begin{abstract}
Sign languages use visual pattern to communicate rather than acoustic patterns that are communication mode in verbal communication. Sign languages being the most structured form of gestures can be considered as the benchmark for systems of gesture recognition. SLR has got its applicability in the areas of appliances control, robot control, interactive learning, industrial machine control, virtual reality, games, simulations etc. apart from its significance for hearing impaired community. The paper aims to present a systematic, robust, reliable, and consistent system for static Pakistani Sign Language (PSL) recognition. The paper is based on empirical evaluation of different classification techniques for SLR. This pragmatic approach leads to a Fuzzy Model (FM) that has shown very high accuracy rate for PSL recognition. Sign languages have inherent uncertainty, so SLR systems demand a classification method that give due consideration to this aspect of uncertainty. This is the reason for selecting fuzzy inference for the proposed SLR system and experimental analysis has proven its suitability for SLR. The meticulous statistical analysis performed for proposed PSL-FM has shown very promising results.
\end{abstract}

Keywords-pattern recognition;fuzzy classifier; sign language; classification.

\section{INTRODUCTION}

The increasing technological developments and advancements in the world of machines are leading to more and more demanding users. Human beings want machines to interact with them in more natural way. The users of the machine wish to have an interaction with machine in the same pattern as they have with their fellow beings. With every progressing day, machines are becoming part of human life, more and more. This intermingling of machines and human is asking for removing the barriers in the communication channels between human and machines and hence researchers are having their focus to make this channel as natural as possible. Human needs speech and gestures to communicate and same is the communication requirements from machines. So these human like modes of communication should be equipped into the machines. The domain of speech as mode of communication is well researched but gestures as mode of human-machine communication still have a lot of room for research. Another motivation behind research in the domain of gesture recognition is the opening of new aspects of communication for the hearing impaired people. For deaf community, Sign Language (SL) as a kind of gestures is the primary communication media. Sign language is considered to be the most structured form of gestures. There are millions of deaf people around the world, who are using sign language for accessing and exchanging information. This is the reason that in recent years, SLR is in focus and researchers came up with variety of solutions. Sign language is the language of visual gestures that are mainly used as a communication tool for deaf community. Sign languages use visual pattern that are used to communicate rather than acoustic patterns that are used in verbal communication. Being most structured form of gestures, Sign language can be taken as benchmark for gesture recognition system. There are many communities in the world, because of having high number of deaf people; the whole community is using gestures instead of verbal communication. Some examples of such communities are Martha's Vineyard Sign Language (USA), Kata Kolok (Bali), Adamorobe Sign Language (Ghana) and Yucatec Maya sign language (Mexico) [15].

Sign languages, just like acoustic languages, have got vast variety. SLs have got diversity in the base language and then these base languages have variety in dialects. Different countries have different signlanguages and then there exist difference of dialects in different regions of same country. Generally, automated SL recognition (SLR) is performed in following phases: image acquisition, gesture segmentation, feature acquisition and classification.

Signer's image is provided as input to Sign Language Recognition system. This input image is then passed onto the segmentation module that segments out the region of interest i.e. hands, face, arm or may be foot, depending upon the scope of the recognition system. This segmented image is then goes for the feature acquisition module. This module extracts required features for the classification. Classification module receives the extracted features andapplies some classification algorithm to give the results of the recognized sign as output.

SL is not internationally uniform; rather it varies from country to county and region to region. Researchers have proposed different SLR systems for different SLs. Some languages are more in focus by researchers such as Chinese 
Sign Language CSL [22,23,24], American Sign Language ASL $[19,20,1,2]$ and Arabic Sign Language ArSL[25,26,27,28]. Unfortunately, Pakistani Sign Language (PSL) could not get its due share in this SLR development. kausar and et. al. [8] has proposed a fuzzy classifier using colored gloves for PSL alphabets recognition. Khalid and et. $\mathrm{Al}$ [16] has made use of data gloves for the recognition of static one handed PSL alphabets. There are approximately nine million people (5\% of total Pakistani population) in Pakistan with hearing impairments [14] and out of these 1.5 million are deaf [14]. Pakistani Sign Language (PSL) is a visual-gestural language, it is a blend of Urdu(national language of Pakistan), and some regional languages. Less than ten thousand Pakistani deaf have attended school [14] because the information exchange methods prevailing in the schools are not sufficiently fulfilling their needs. Bringing PSL in modern technologies using SLR can improve the lives of deaf community in many aspects. SLR is a complex domain and has many inherent challenges with respect to region of interest segmentation, signer's environment, descriptor's invariance, sign articulation, variation in sign dictionary, selection and extraction of representative sign descriptors, correct, efficient and reliable classification.

The paper presents a reliable and efficient method for PSLR. The proposed method uses static one-handed signs. Classification method is the main contribution of the paper,

\section{RELATED WORK}

The domain of sign language recognition can be used as benchmark in many other gesture recognition systems. This diverse applicability of the domain has compelled the focus of many researchers towards it. This focus has led to the immense and valuable contribution.

Multiple cameras have been used by Elons et al. [5] and Dreuw et al. [1] for recognition of SL. Two different viewing angles have been utilized by Elons et al. to generate image features using pulse coupled neural network for recognition of American Sign Language. Special angle setting is required for mounting cameras to get 3 dimensional features. So system requires multiple cameras and complex angle adjustments for accurate recognition. Priyal et al. have utilized Krawtchouk moment features for recognition of static hand sign [9] with a claim of signer independence and incorporation of camera angle variation. Li. et al. have recognized Chinese sign language using portable accelerometer and surface electromyography sensors [3]. High accuracy rates are claimed using hand shape, movement and orientation. Data gloves have been used byShijian et al. [10] and Tan et al. [11] for SLR. Data gloves raise the accuracy rate but system's dependence on external gadgetry is not appreciated and accepted widely. Colored and marked gloves have been used by Akmeliawati [12] and kausar et al. [13 for SLR. Prime points of hand are marked with special colors; this marking has made the recognition easier. The size of dictionary of signs for SLR systems is quite small in many cases. Al-Alali et al. [6] proposed a system for only four signs. Wassnerr et al. developed a system that was for recognition of only two French signs. Zafrullah et al. [2] have proposed American Sign
Language recognition system to recognize only six signs. Ershaed et al. [6], Brashear et al. [2], Xing et al. [4] and Helen et al.[13] have recognized SL by utilizing depth cameras. The depth cameras are quite costly.

This section has presented few contributions in SLR domain. It is observed that different proposed methods have their own strengths and weaknesses. The proposed method has undertaken all these weaknesses of SLR systems into consideration and tried to reduce these significantly in the proposed system.

\section{Methodology}

The paper presents PSL recognition system using a novel method. Signer's image is provided as input to the system. This input image then processed by the segmentation module. The segmentation module segments the signer's hand. The segmented hand is then transformed into one dimensional signature. This signature is then parameterized to obtain polynomial coefficients. These polynomial coefficients are then fed into the fuzzy classifier as feature vector. Classifier then classifies the sign and displays the output. Rest of the paper is providing the details of these modules.

System initiates with the input image of signer. The image frame only has one hand. Signer has to wear full sleeves shirt. Signer's background should be non-occluded. These constraints make the segmentation process, simple and accurate. The system does not require expensive and cumbersome external gadgetry for input; rather it purely relies on visual input. Ordinary 2-D camera is used for image capture; range cameras have associated barriers of computational complexity, cost, and special experimental environment. The proposed image capturing makes the system, cost effective and more adoptable. The captured signer image is then passed to the segmentation module. K-means clustering is used for the segmentation of hand. K-means clustering is used to segment out skin color pixels from the background. To improve the segmentation, morphological operations are performed on the segmented hand. The output of the segmentation module is the binary image of the signer's hand. This binary hand image is then further processed for extraction of sign descriptor.

\section{A. Sign Descriptors}

After the conversion to binary image, the image is processed further for extraction of sign descriptors. Signature based features are used as descriptors. The descriptor used, is very robust and efficient. The descriptor set is quite compact but results are very promising. The binary image of hand is transformed into one dimensional shape signature. These signatures are then used to approximate the parameters of the signatures. Different types of parameterizations are experimented for PSL recognition. Gaussian, Polynomial, exponential and sine parameterization is applied on the shape signature. Different numbers of coefficients for each parametric class are tried out to check their appropriateness for SLR. These coefficients are then used as sign descriptor for classification. Polynomial parameterization gives the best 
results[17]. The process of PSL sign descriptor extraction can be summarized with the following figure.

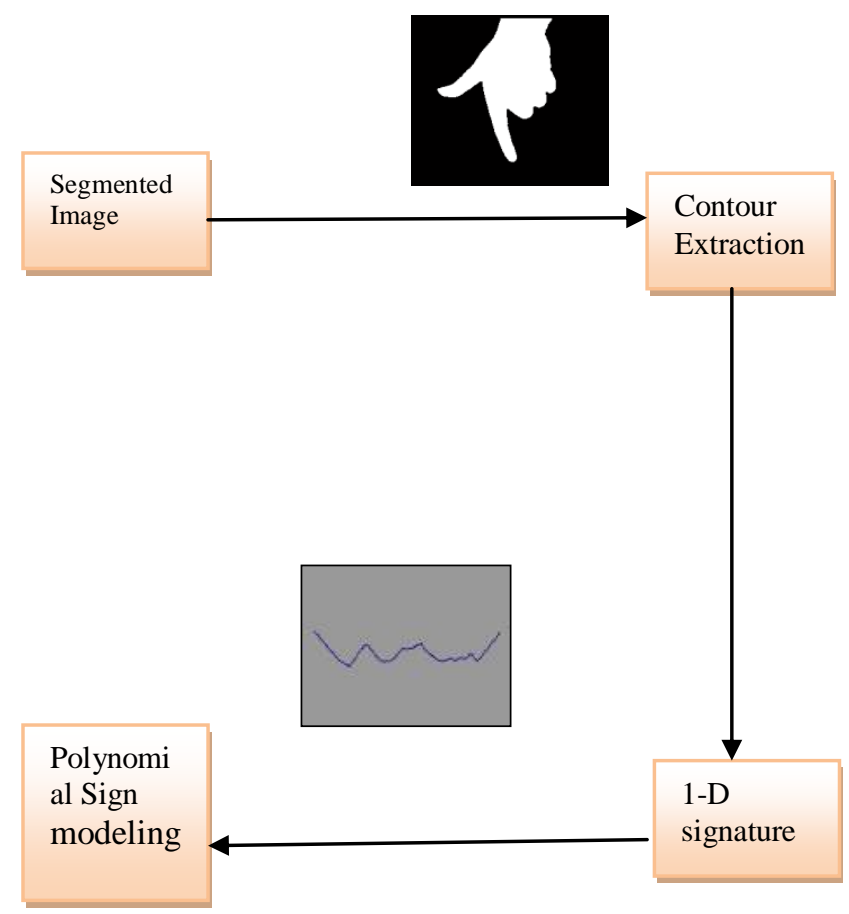

Figure 1: Sign Descriptor Extraction

$i$.

Sign Contour Extraction

For transforming sign to its corresponding 1-D signature, centroid distance is a very popular method. For obtaining centroid, segmented binary image $\underset{\mathrm{E}}{\mathrm{C}}(\mathrm{x}, \mathrm{y})$ is obtained as a result of segmentation. Let $T$ is the original image having hand sign, where $\mathrm{T}(\mathrm{x}, \mathrm{y})$ is the point at $(\mathrm{x}, \mathrm{y})$ location in the original image. Transformation of $\mathrm{T}(\mathrm{xi}, \mathrm{yi}) \rightarrow \underset{\mathrm{E}}{\mathrm{E}}\left(\mathrm{x}_{\mathrm{i}}, \mathrm{y}_{\mathrm{i}}\right)$ in such a way that $\Phi(x i, y i) \in d$ would be assigned value 1 in the transformed image $\breve{E}$ and rest treated as background. So binary segmented image Ȩ is:

$$
\begin{aligned}
& \breve{\mathrm{E}}(\mathrm{x}, \mathrm{y})=1 \text { iff }(\mathrm{x}, \mathrm{y}) \in \mathrm{d} \\
& \breve{\mathrm{E}}(\mathrm{x}, \mathrm{y})=0 \text { otherwise }
\end{aligned}
$$

Where, ' $\mathrm{d}$ ' is the domain of binary segmented image of sign. Centroid (Ḉ(x), Ḉ(y)) of the hand sign is the center of mass $\forall \ddot{v}_{\mathrm{i}} \in \mathrm{d}$. where $\tilde{v}_{\mathrm{i}}=\underset{\mathrm{E}}{\mathrm{E}}\left(\mathrm{x}_{\mathrm{i}}, \mathrm{y}_{\mathrm{i}}\right)$. It can be obtained as follows:

$$
\begin{aligned}
& C^{\prime}(x)=\frac{1}{N} \sum_{i=1}^{N} x_{i} \\
& C^{\prime}(y)=\frac{1}{N} \sum_{i=1}^{N} y_{i}
\end{aligned}
$$

Where $\mathrm{N}$, is the total number of points in the segmented sign i.e. $\mathrm{N}=\sum \ddot{v}_{i} \in \breve{E} \cap d$. So only point $\ddot{v}_{i-\rightarrow}$ to be considered is, that holds following property:

$$
\left(x_{i}, y_{i}\right) \mid \breve{E}\left(x_{i}, y_{i}\right)=1
$$

After obtaining (Ḉ(x), Ḉ(y)), 1-Dimensional signature $\lambda_{i}$ needs to be extracted. Hand sign contour points are required for this. Contour points are extracted by taking $\forall \ddot{v}_{i} \in(\breve{E} \cap$ d) $\cap \mathrm{R}\left(\ddot{v}_{i}\right) \in \sim q$. Where $\mathrm{R}\left(\ddot{v}_{i}\right)$ is the neighbor of $\ddot{v}_{i}$.

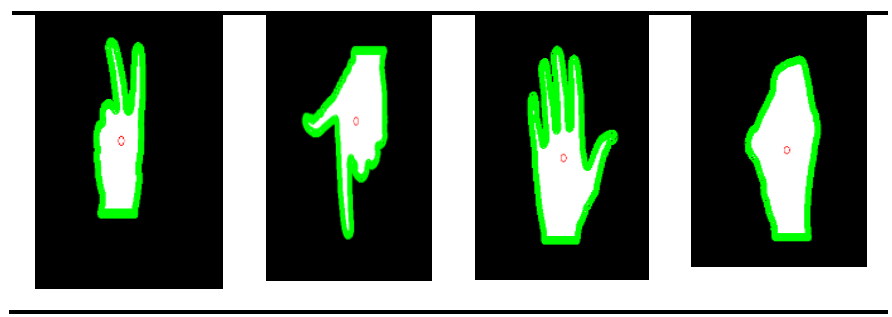

Figure 2: plot of centroid poin(red)t and contour points(green)

ii.

One dimensional sign Signature

Once the contour points of hand are calculated, $\lambda_{i}$ is obtained using centroid distance Let the contour points of the hand sign are represented with a vector $\dot{\rho}=$

$\left[\left(\dot{\rho}_{1}(x), \dot{\rho}_{1}(y)\right),\left(\dot{\rho}_{2}(x), \dot{\rho}_{2}(y)\right), \ldots \ldots,\left(\dot{\rho}_{n}(x), \dot{\rho}_{n}(y)\right)\right] \cdot \lambda_{i}$ can be obtained as: $\lambda_{i}=\left|\dot{\rho}_{i}(x)-\mathcal{C}(x), \dot{\rho}_{i}(y)-\zeta \zeta(y)\right|$ $\lambda_{i}$ is the $\mathrm{i}^{\text {th }}$ distance point between $\mathrm{i}^{\text {th }}$ contour point and centroid of hand sign. The centroid point of the hand sign is

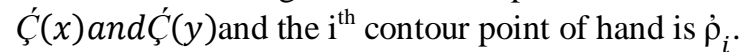

For time efficiency, sampling of contour points is performed before extracting the shape signature. To incorporate the size variability of hands, adaptive step size for taking samples from contours is used.

Centroid distance based signature is translation, rotation and scaling invariant.
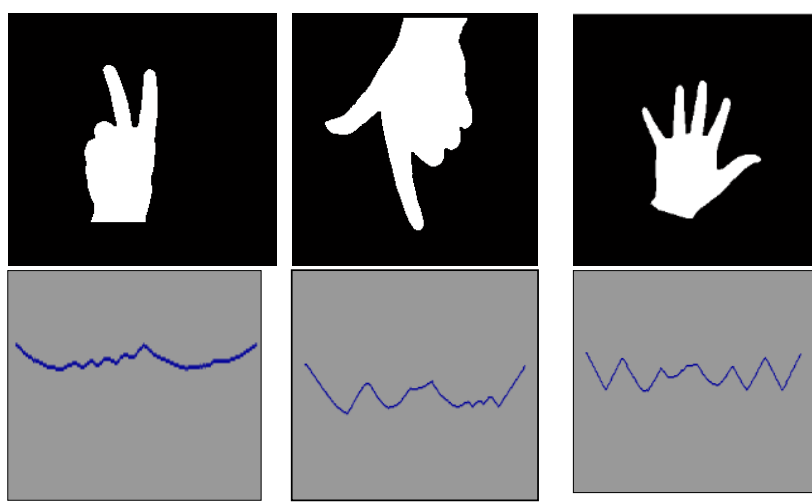

Figure 3: 1-Dimensional signatures for signs of PSL

\section{B. PSL Sign Model}

Mathematical modeling and parameterization is very new concept in the area of SLR. Polynomial modeling of PSL has been empirically proven best among other competitive models [17]. Fitting a polynomial mathematical function of a particular degree to the sign signature is termed here as 


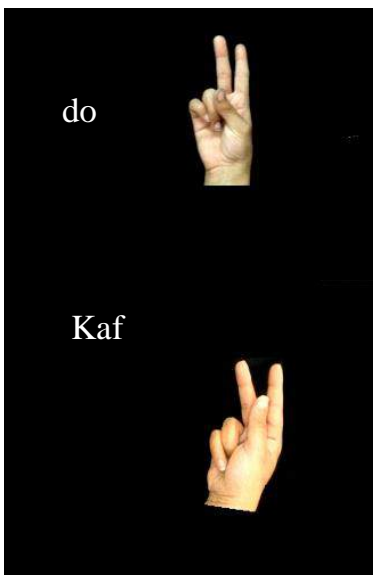

Figure 4: uncertainty in signs

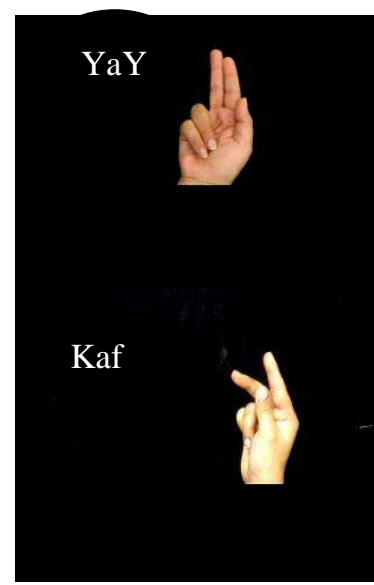

polynomial parameterization. A single polynomial function is fitted to each sign signature while capturing the overall trend of the points of sign signature.

Sign signature is the interpolation of the data directly acquired from the sign. This interpolation gives one dimensional description of the data and this description can be directly used as feature vector say by transforming it into Fourier domain. But this transformation overhead and then such a big feature vector can no way lead to an efficient and cost effective method for SLR. PSL sign model deals with these issues. Instead of interpolation, the method goes for fitting a general function $\dot{\omega}(\mathrm{x})$ approach. The fitting of function $\dot{\omega}(\mathrm{x})$ involves, empirical evaluation of number of coefficients for a function class, tuning these coefficients to get the 'best' representation of the sign signature. 'Best' representation is evaluated using approach of least square residual. Let $\delta$ be a vector having size $=\mathrm{n}, \quad=$ $\left[\delta_{1}, \delta_{2}, \delta_{3} \ldots \ldots, \delta_{n}\right]$, and let $\dot{\omega}$ be a vector of size $n$ such that $\dot{\varphi}$ $=\left[\dot{\omega}\left(x_{1}\right), \dot{\omega}\left(x_{2}\right), \dot{\omega}\left(x_{3}\right) \ldots \ldots, \dot{\omega}\left(x_{n}\right)\right]$. Error minimization can be defined as:

$$
\begin{gathered}
\dot{\varepsilon}=\sum_{i}^{n} f_{i}^{2} \\
f_{i}^{2}=\left(\alpha_{i}-\dot{\omega}\left(\mathrm{x}_{\mathrm{i}}\right)\right)^{2}
\end{gathered}
$$

The total number of data points in the sig nature is $n, \mathrm{i}^{\text {th }}$ actual contour point is $\alpha_{i}$ and the value approximated through mathematical model is.$\dot{\varphi}\left(\mathrm{x}_{\mathrm{i}}\right)$.

$\grave{\varphi}\left(x_{i}\right)$ with minimum value for $\varepsilon$ function is selected. Polynomial function can be defined as:

$$
\dot{\omega}\left(x_{i}\right)=\rho_{0}+\sum_{j=1}^{n} \rho_{j} x_{i}^{j}
$$

Order of polynomial function is denoted with $\mathrm{n}$, for the proposed function, $n$ goes from $1 \rightarrow 9$. ith coefficient of the polynomial function is represented with gi. So a polynomialfunction of degree nine would actually return 10
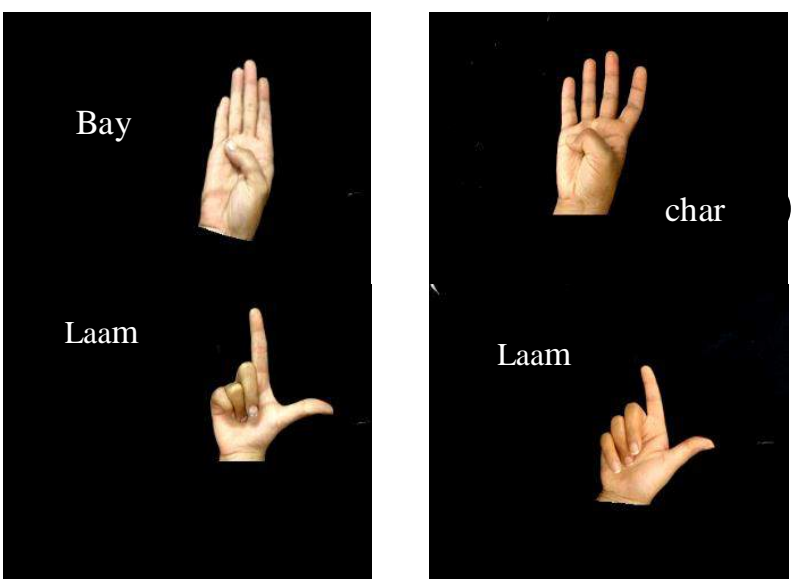

analyzed in analysis section.

coefficients i.e. $0 \rightarrow 9$. We need to get such values of $g$ that give minimum

value for $\dot{\varepsilon}$ for $\hat{\omega}\left(\mathrm{x}_{\mathrm{i}}\right)$. Experimentation is also performed to explore the appropriate numbers of coefficient. The polynomial mathematical model for PSL is static, explicit, discrete, deterministic and inductive.

\section{Classification}

Soft classifiers are suitable for the problem domains, where working with crisp boundaries for classification is not very effective. The inherent uncertainty in certain problem domain pursues to opt for a classifier that is having a soft decision capability instead of the hard one. The soft classifier respects the uncertainty and provides a flexibility in the decision making process.

\section{i. Uncertainty}

The special consideration for uncertainty is required for SLR. The sign itself have a lot of uncertainty. Human mind is capable of dealing very well with the uncertainty. Human mind does not work on crisp boundaries for recognizing sign i.e. it does not need to define the accurate angles of finger or precise level of tilt in the fingers; rather human brain works well with qualitative term. For instance two signs of PSL naming "do" and "yay" are different just on the basis of the position of thumb and finger alignment, if we try to find the exact boundary between these two signs it would be difficult. Another such example is "bay" and "char".

The same sign posed by different signers would definitely possess some variations and if the signs are strictly defined in the terms of their Examples of straightness or tiltness of the fingers then their slightly rotated variants would be difficult to get accommodated within the hard and crisp boundaries of conventional statistical tools.

Expectations from the conventional classification tools to deal with such uncertainty normally end up with disappointment. As traditional statistical classification tools does not respect the importance of the uncertainty and these tools lack the capability of dealing the qualitative term in a desired manner. These tools tend to transform the qualitative 
terms to the rigid and hard quantitative terms. These are the reasons to look for some unconventional classification tool that incorporates the inherent uncertainty of sign language. So fuzzy classification can be very suitable choice in this regard. This claim for suitability is proved and analyzed in analysis section.

\section{ii. Fuzzy Classification}

Fuzzy classification is based on fuzzy inference process. Fuzzy inference process is basically mapping of input to output, and this mapping is materialized by fuzzy logic. There are three main steps in fuzzy inference process i.e. fuzzification of inputs, applying inference rules and then defuzzification of output.

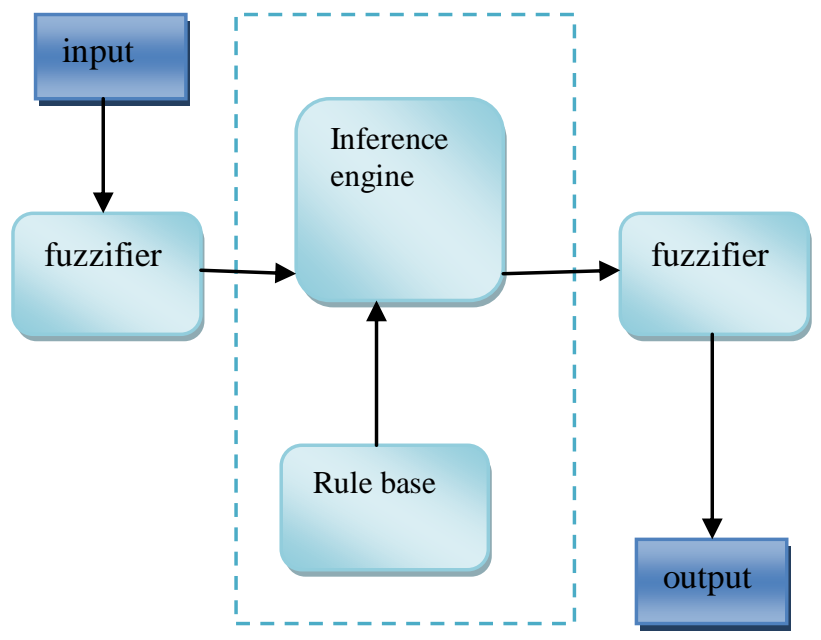

Figure 5 Fuzzy process

The first step for fuzzy inference process is to fuzzify the input variables. Input is always a crisp value within a universe of discourse and that input is fuzzified by utilizing the concept of membership function. A curve that is mapping every point from input space to corresponding membership value between 0 and 1 , is called membership function. The output of this step is a fuzzy value that is transformed from the crisp input into the degree of membership for all the qualifying membership functions required by the fuzzy rules. This process is repeated for all the inputs. The degree of membership always ranges from $0 \rightarrow 1$. For the paper Gaussian membership function is used for the input and triangular function for output membership function.

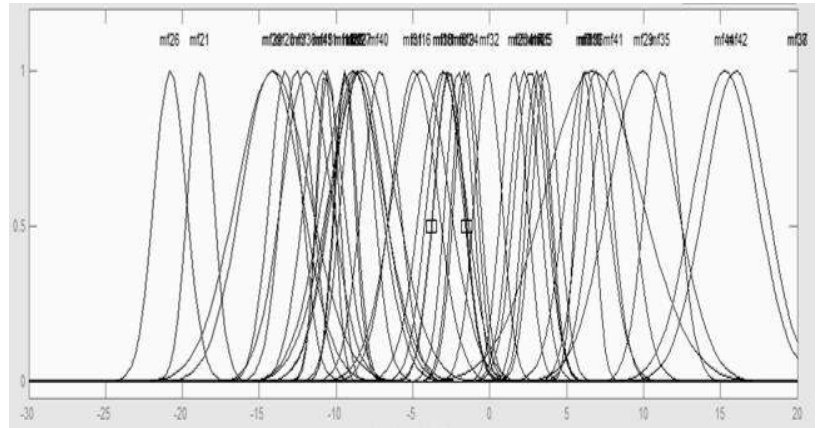

(a)

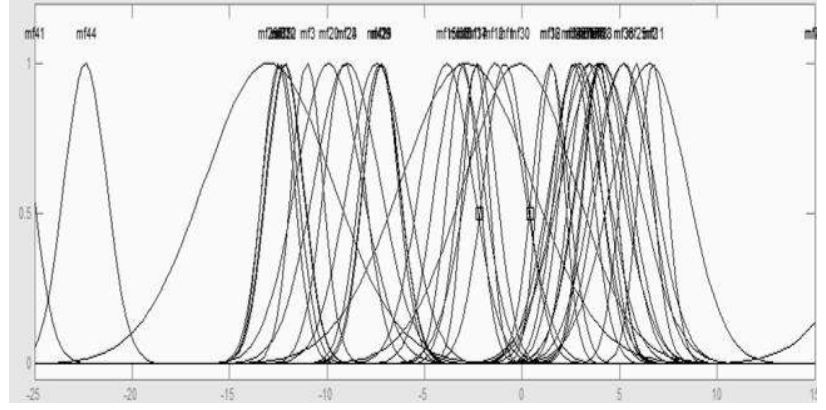

(b)

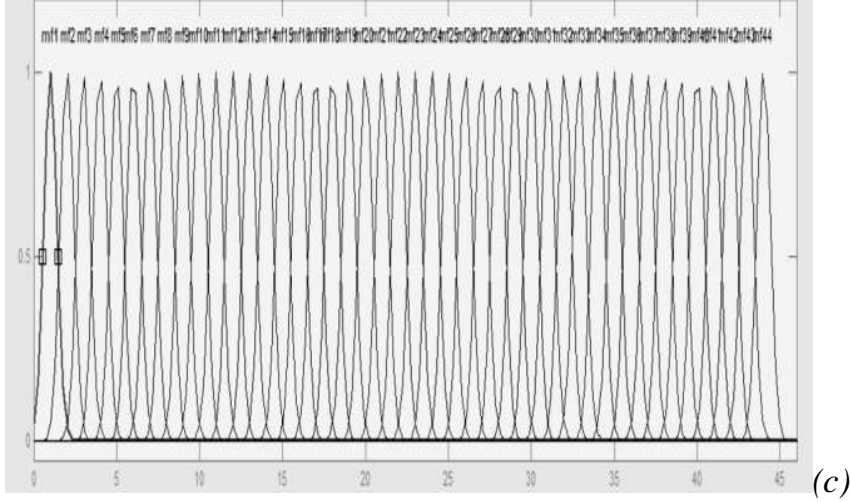

Figure 6 : membership functions (a) $Q_{-} 0$ (b) $Q_{-} 1$ (c) output

The fuzzy logic is comprised of if-then statements. These if-then statements used for fuzzy inference are called fuzzy rules. These rules are applied once the input values are fuzzified. Some examples of if- then rules can be as follows.

There are three sub-steps of applying fuzzy rules; Applying fuzzy operator, Applying implication method and aggregation of outputs. If the antecedent part of the fuzzy rule has multiple terms then there is a need to apply fuzzy operator. The value of antecedent obtained after applying fuzzy operator, is then used by the implication method to get the shape of the consequent of each fuzzy rule. Consequent is a fuzzy set that is shaped by a corresponding membership function. The output of the implication process is the reshaped consequent using the consequent's membership function based on a single number. 
After applying implication method for reshaping the consequent membership function for each rule, the aggregation of the output needs to be performed. The aggregation is the process of combining the fuzzy sets of all the rules in a way to give a single fuzzy set to make the decision. The input to the defuzzification step is the aggregate fuzzy set. This fuzzy set cannot be directly evaluated to make a decision as it possess a range of values. So defuzzifier resolves the fuzzy set up to single number. There are many methods that can be applied for defuzzification on the aggregate fuzzy set. This number is the output of the complete fuzzy inference process.

\section{RESULTS}

Standard data set is not available for PSL. So self-developed data is being used for this paper. Dataset is named as Dataset PSL Table 1.

TABLE1: DATASETPSL

\begin{tabular}{|l|l|l|l|l|}
\hline Dataset & $\begin{array}{l}\text { Number } \\
\text { of Signs }\end{array}$ & $\begin{array}{l}\text { Number } \\
\text { of Signers }\end{array}$ & $\begin{array}{l}\text { test } \\
\text { set }\end{array}$ & $\begin{array}{l}\text { training } \\
\text { set }\end{array}$ \\
\hline Dataset $_{\mathrm{PSL}}$ & 44 & 10 & 279 & 387 \\
\hline
\end{tabular}

PSL recognition is quite challenging, as it has many visually similar signs. There are many examples in Dataset PSL, where same sign having different orientation is representing different sign. e.g. "daal" and "ain", "zaal" and "ghain" and "zaal" are such examples. Similarly there are other examples in PSL, where signs are visually quite similar to each other. Such as" tuay" and "zuay", "dal' and "tuay", "aliph" and "tay", "ain" and "ghain" etc (Figure. 8). Complexity of problem is added due to the aspect of inherent uncertainty of SL. These challenges are well incorporated in proposed method.
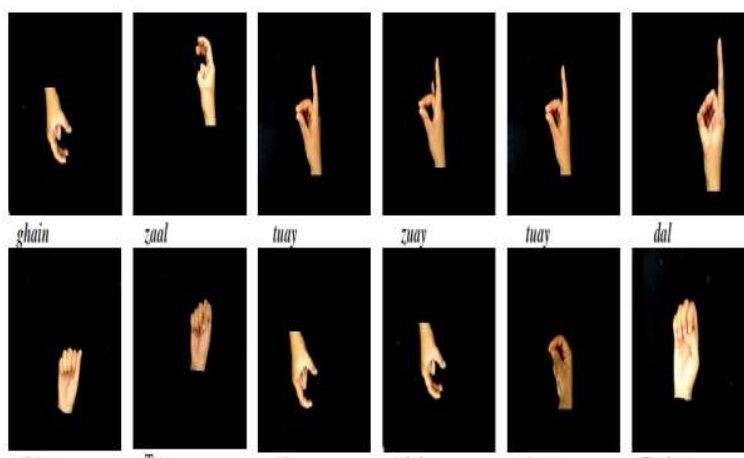

Figure 7: visually similar signs

The proposed method named as PSL Fuzzy Model (PSL-FM), is compared with other alternative models. The following table is showing the comparison of the proposed classifier (PSL-FC) with other classifiers while using the
If $\left(\rho_{0}\right.$ is in range 1 and $\rho_{1}$ is in range 1 and $\rho_{2}$ is in range 1 and $\rho_{3}$ is in range 1 and $\rho_{4}$ is in range 1 and $\rho_{5}$ is in range 1 and $\rho_{6}$ is in range 1 and $\rho_{7}$ is in range 1 and $\rho_{8}$ is in range 1 and $\rho_{9}$ is in range 1 )

Thenaliph

If $\rho_{0}$ is in range 2 and $\rho_{1}$ is in range 2 and $\rho_{2}$ is in range 2 and $\rho_{3}$ is in range 2 and $\rho_{4}$ is in range 2 and $\rho_{5}$ is in range 2 and $\rho_{6}$ is in range 2 and $\rho_{7}$ is in range 2 and $\rho_{8}$ is in range 2 and $\rho_{9}$ is in range2)

Then bay

If $\left(\rho_{0}\right.$ is in range 3 and $\rho_{1}$ is in range 3 and $\rho_{2}$ is in range 3 and $\rho_{3}$ is in range 3 and $\rho_{4}$ is in range 3 and $\rho_{5}$ is in range 3 and $\rho_{6}$ is in range 3 and $\rho_{7}$ is in range 3 and $\rho_{8}$ is in range 3 and $\rho_{9}$ is in range 3 )

Then pay

proposed sign model as feature set for all of the classifiers. Support Vector Machine is a potential candidate to get comparison with but it is a binary classifier and 44-class problem compel to have a hierarchal implementation of SVM. 44 level hierarchal model of SVM needs too many computational and time resources. This is why SVM is not giving high accuracy as expected for PSL. One-versus-all method is followed here for multi-class SVM implementation. So for a 44-class problem, the SVM considerably loses its strength of classification.

TABLE 2: COMPARISON OF PROPOSED CLASSIFIER (PSL-FC) WITH OTHER CLASSIFIERS

\begin{tabular}{lll}
\hline Classifier & \multicolumn{1}{c}{$f$} & Accuracy \\
\hline 3-NN & 0.69 & $74.8 \%$ \\
5-NN & 0.63 & $63.4 \%$ \\
7-NN & 0.58 & $58.06 \%$ \\
SVM & 0.64 & $68.1 \%$ \\
Naïve Bayes & 0.35 & $35.4 \%$ \\
PSL-FM & $\mathbf{0 . 9 8}$ & $\mathbf{9 8 . 2 \%}$ \\
\hline
\end{tabular}

True positive, false negative and false positive rate is considered to be a very effective gauge for the performance of any classification system. 
TABLE3 COMPARATIVE ANALYSIS OF DIFFERENT PSL RECOGNITION SYSTEMS WITH PROPOSED MODEL

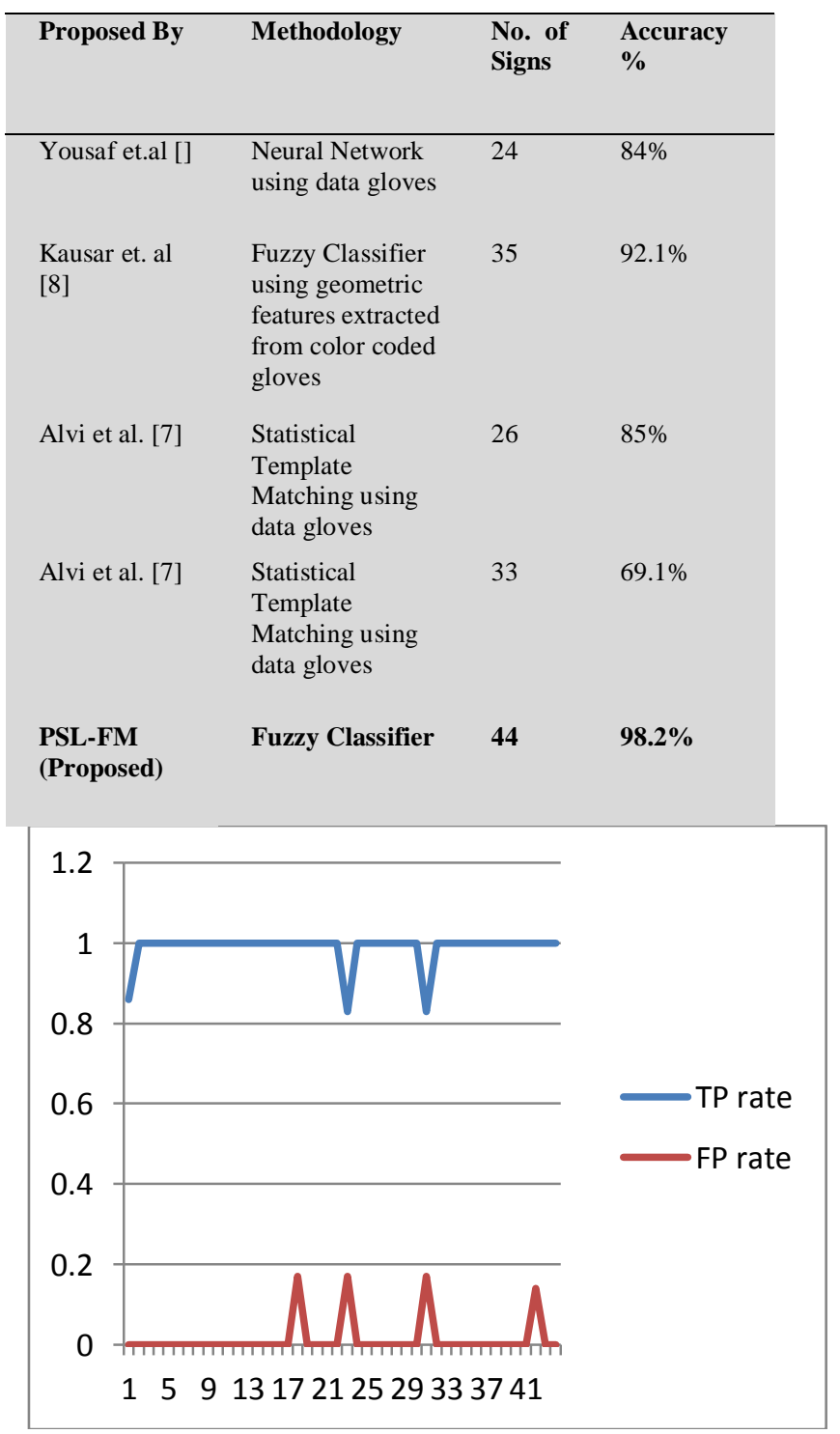

Figure 8: True positive and False Positive rate analysis

Recall and precision are established statistical analysis measure. These are defined in terms of true positive and false positive. Recall and precision defines F measure. F measure is used to analyze the PSL-FM.

$F=2 *($ Precision $*$ recall $) /($ Precision + recall $)$

Precision $=$ true positive/ $($ true positive + false positive $)$

Recall $=$ true positive/ $($ true positive + false negative $)$

Figure 9 shows the analysis of PSL-FM using recall, precision and $\mathrm{F}$ measure for each sign of the dataset. The Figure 10shows the comparative analysis of different PSL recognition systems. The comparison reinforces the fact about the high accuracy achievement of the proposed system. The proposed system is developed for bigger dataset as compared to the other PSL recognition systems; even then its accuracy rate is much higher than others.

There is very small part of research contribution towards the Pakistani sign language recognition system. Even after very exhaustive search for such publications, the above mentioned only four researches could be found. The proposed system is far better than these systems in term of accuracy and number of sign dictionary.

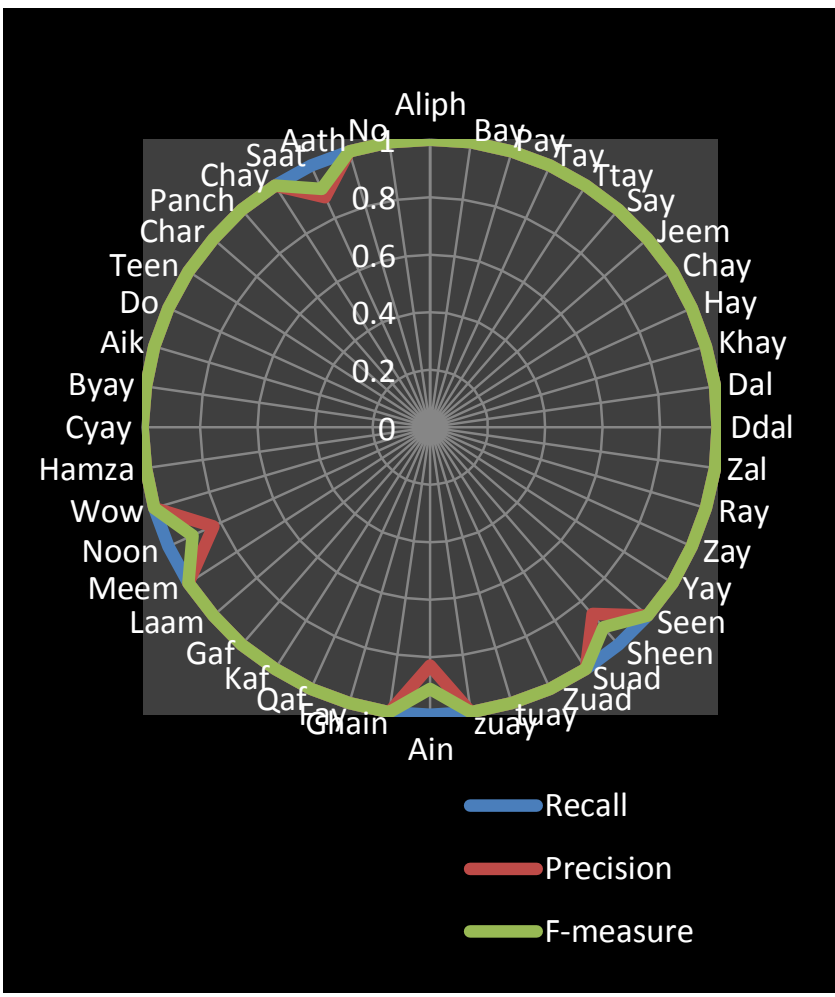

Figure 9: Recall precision and F measure analysis using Radar plot of Dataset combined

Apart from accuracy analysis, Comparative time analysis is also performed for PSL-FM. This time analysis is just a way of comparing the time efficiency of different alternatives for PSL recognition. The proposed methodology does not involve any training so training time is zero. SVM is the most costly classifier in terms of training time as it needs training time once for the training set and once for the support vector training time. The recognition of a sign is also not taking considerable time. So not only the accuracy, the time efficiency of PSL-FM is also quite high if compared to other competitive techniques. 


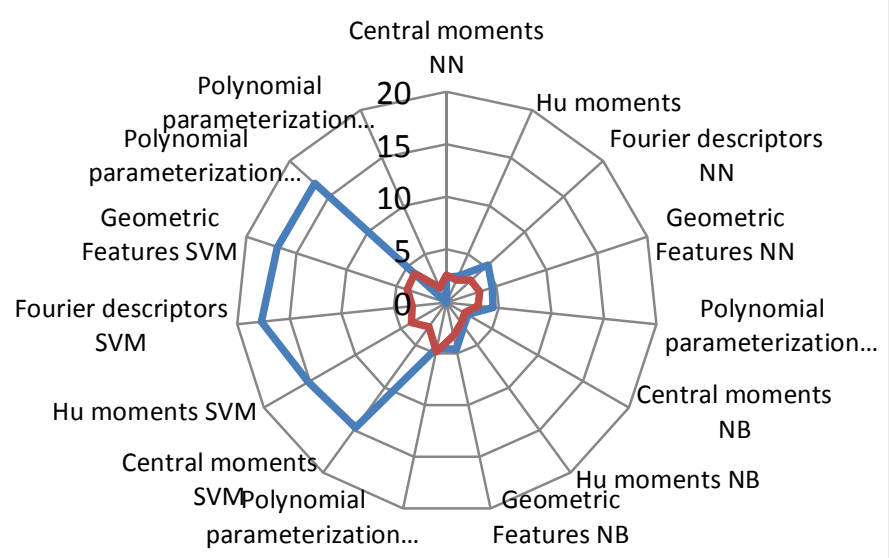

_Training Time(387 examples) scaled down by a factor of 100

Test time per Sign

Figure 10: Time analysis of proposed model

The multi-aspect analysis of PSL-FM has shown that the proposed methodology has outperformed many other classification systems. The system can be considered as a framework that can get tailored for different gesture recognition systems.

\section{CONCLUSION}

The paper presents a signer-independent robust and efficient classification method for PSL. The proposed classification method can be used as sort of a framework that can get tuned for other sign languages by empirical analysis for readjustment of fuzzy rules. Parameterization of shape signatures as sign descriptor is presented. Main contribution of the paper is on classification module, fuzzy classifier is proposed. The paper is an effort to contribute in the services area for a special group of humanity i.e. Deaf society. This is a research area that should have ongoing research. The proposed methodology has produced very high accuracy rate for recognition of PSL. The approach of mathematical modeling can be further extended to the dynamic sign language recognition. The dataset for PSL can be extended further for future research.

\section{REFERENCES}

[1] Philippe Dreuw, Pascal Steingrube, Thomas Deselaers, Hermann Ney. Smoothed Disparity Maps for Continuous American Sign Language Recognition. In: Pattern Recognition and Image Analysis LECT NOTES COMPUT SC 2009; 5524: 24-31.

[2] Zafrulla, H. Brashear, T. Starner, H. Hamilton, and P. Presti. American sign language recognition with the kinect. In: Proceedings of the 13th International Conference on Multimodal Interfaces, ICMI '11, 2011; New York, NY, USA. Pp. 279-286.

[3] Yun Li, Xiang Chen, Xu Zhang, Kongqiao Wang, and Z. Jane Wang.. A Sign-Component-Based Framework for Chinese Sign Language Recognition Using Accelerometer and semg Data. In: IEEE T BIO-MED ENG 2012; 59.

[4] Xing Guo, Zhenyu Lu, RongbinXu, ZhengyiLiu ,Jianguo Wu. Big-screen text input system based on gesture recognition. In: Proceedings of the 2nd International Conference On Systems Engineering and Modeling (ICSEM-13), 2013; Paris; France.

[5] A. Samir Elons, MagdyAbull-ela, M.F. Tolba . A proposed PCNN features quality optimization technique for pose-invariant 3D Arabic sign language recognition. In: APPL SOFT COMPUT April 2013;13: 1646-1660.

[6] Ershaed, I. Al-Alali, N. Khasawneh, and M. Fraiwan. An arabic sign language computer interface using the xboxkinect. In: Annual Undergraduate Research Conf. On Applied Computing May 4-5, 2011; Dubai, UAE.

[7] Aleem Khalid Alvi, M. Yousuf Bin Azhar, MehmoodUsman, SulemanMumtaz, Sameer Rafiq, raziurRehman, Israr Ahmed. Pakistan Sign Language Recognition Using Statistical Template Matching. In: World Academy of Science, Engineering and Technology , 2005; 3 .

[8] SumairaKausar, M. Younus,Javed, Shaleeza,Sohail. Recognition of gestures in Pakistani sign language using fuzzy classifier. In: 8th conference on Signal processing, computational geometry and artificial vision 2008; Rhodes, Greece: pp. 101-105

[9] S. PadamPriyal, Prabin Kumar Bora. A robust static hand gesture recognition system using geometry based normalizations and Krawtchouk moments. In: Pattern RecognitionLECT NOTES COMPUT SC; August 2013; 46: 2202-2219.

[10] Xushijian, Xujie, Tang yijun, Shenlikai. Design of the Portable Gloves for Sign Language Recognition. In: Advances in Computer Science and Education Applications Communications in Computer and Information Science; 2011; 202:171-177.

[11] Tan TianSwee, A.K. Ariff, S.-H. Salleh, Siew Kean Seng, Leong SengHuat. Wireless data gloves Malay sign language recognition system. In: proceeding of 6th International Conference on Information, Communications \& Signal Processing 2007.

[12] RiniAkmeliawati, Melanie Po-LeenOoi and Ye Chow Kuang. Real-Time Malaysian Sign Language Translation using Colour Segmentation and Neural Network. In: IMTC 2007 Instrumentation and Measurement Technology Conference 1-3 May 2007; Warsaw, Poland; 2007. 
[13] Helen Cooper, Eng-Jon Ong, Nicolas Pugeault, Richard Bowden. Sign Language Recognition using Sub-Units", In:J MACH LEARN RES 2012; 13: 2205-2231.

[14] Annual report 2013 by Family Educational Services Foundation (FESF).

[15] Disabled World: Sign Language and Deaf Communication Methods and Information Http://www.disabled-world.com/disability/types/hearing/comm unication/. Accessed on: 12.12.2013.

[16] Disabled World: Sign Language and Deaf Communication Methods and Information

Http://www.disabled-world.com/disability/types/ hearing/communication/. Accessed on: 12.12.2013.

[17] Sumairakausar, Javed, Y., samabiatehsin, Almas Anjum, “A Novel Mathematical Modeling and Parameterization for Sign Language Classification". International Journal of Pattern Recognition and Artificial Intelligence, Vol 30 (5), pp. 1-21, Apr. 2016.

\section{AUTHOR PROFILE}

Sumaira Kausar is Asst. Professor at Bahria University in Department of Computer Science. Her major areas of interest are Computer Vision and Pattern Recognition.

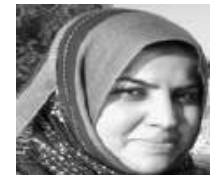

Samabia Tehsin is Asst. professor at Bahria University in Department of Computer Science. Her major areas of interest are Document Analysis, Computer Vision, Image Processing and Pattern Recognition.

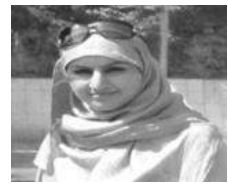

Muhammad Younus Javed is Professor at Hitec university, Pakistan. His major areas of specialization are Adaptive Systems, Parallel Computing, Computer Vision and Algorithmics.

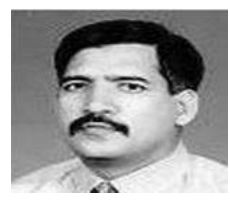

(C2016 by the author(s); licensee Empirical Research Press Ltd. United Kingdom. This is an open access article distributed under the terms and conditions of the Creative Commons by Attribution (CC-BY) license. (http://creativecommons.org/licenses/by/4.0/). 